Bull. Austral. Math. Soc.

Vor. 49 (1994) [277-292]

\title{
GLOBAL ATTRACTOR FOR THE CAHN-HILLIARD SYSTEM
}

\author{
Jan W. Cholewa and Tomasz Dlotko
}

The Cahn-Hilliard system, a natural extension of the single Cahn-Hilliard equation in the case of multicomponent alloys, will be shown to generate a dissipative semigroup on the phase space $\mathcal{H}=\left[H^{2}(\Omega)\right]^{m}$. Following Hale's ideas and based on the existence and form of the Lyepunov functional, our main result will be the existence of a global attractor on a subset of $\mathcal{H}$. New difficulties specific to the system case make our problem interesting.

\section{INTRODUCTION AND NOTATION}

This paper justifies the existence of a global attractor for the system of CahnHilliard equations

$$
w_{t}=\Delta\left[-\Gamma \Delta w+\nabla_{w} \Phi(w)\right] \quad(t, x) \in \mathbb{R}^{+} \times \Omega
$$

where $w: \mathbb{R}^{+} \times \Omega \rightarrow \mathbb{R}^{m}, \Gamma=\left[\Gamma_{i j}\right] \in \mathbb{R}^{m \times m}$ is a symmetric and positive definite matrix, $\Omega$ is a bounded domain in $\mathbb{R}^{n}(n \leqslant 3)$ having $C^{4}$ regular boundary $\partial \Omega$ and $\Phi: \mathbb{R}^{m} \rightarrow \mathbb{R}$ has locally Lipschitz continuous partial derivatives up to the third order. The system (1) is considered with homogeneous boundary conditions

$$
\left.\nabla_{\boldsymbol{x}} w N\right|_{x \in \theta \Omega}=\left.\nabla_{x}(\Delta w) N\right|_{x \in \theta \Omega}=0
$$

(here $\nabla_{x} w=\left[\left(\partial w_{i}\right) /\left(\partial x_{j}\right)\right]$ is a gradient $m \times n$ matrix, while $N$ denotes an outward normal vector to $\partial \Omega$ ) and with an initial condition

$$
w(0, x)=w_{0}(x)
$$

for a vector function $w_{0}$ from the product space $\left[H^{2}(\Omega)\right]^{m}$, satisfying the compatibility condition $\nabla_{x} w_{0} N=0$ on $\partial \Omega$.

For $m=1$, the problem (1)-(3) reduces to the original Cahn-Hilliard model, studied by many authors (for example $[1,10,2]$ ), describing decomposition of a binary alloy. In general the system (1)-(3) is proposed as a phase separation model in the

Received 19 April 1993

The authors are grateful to Dr. Keng-Huat Kwek for pointing to the problem considered in this paper. The paper was supported by the State Committee for Scientific Research Grant No. PB 0021 P3/93/05.

Copyright Clearance Centre, Inc. Serial-fee code: 0004-9729/84 \$A2.00+0.00. 
case where the alloy consists of $m+1$ components (decomposition of a multicomponent alloy). The derivation of (1)-(3), as well as many theoretical results concerning this problem, can be found for example in [3] or [4]. However, based on the single CahnHilliard equation considerations of $[\mathbf{1 0}]$ and $[\mathbf{2}]$ it is possible to investigate the dynamics of (1)-(3) from the point of view of dissipative systems presented in [5] and general theory given in [6].

Since we are dealing with a system we use mostly matrix (vector) notation and calculus, writing the components directly only when it is needed for the clarity of local calculations. The components of a vector $s \in \mathbb{R}^{d}$ are written as $s_{i}$, whereas partial derivatives are denoted in the standard way, with explicitly given variable with respect to which differentiation is taken; for example the components of the nonlinear term $\nabla_{w} \Phi(w)$ are written simply as $\frac{\partial \Phi}{\partial w_{i}}(w)$. The differential operators $\Delta, \nabla$ are understood to be taken with respect to spatial variable $x$; otherwise we always indicate the variable explicitly in the index, as in the case of $\nabla_{w} \Phi(w)$. For a matrix $B, \operatorname{tr}(B)$ is the sum of the diagonal elements of $B$ and $B^{T}$ is the transposed matrix of $B$; also by the integral (or derivative) of a matrix we understand the matrix of the integrals (derivatives) of its elements. Notation of Sobolev spaces is standard [10,11]. Product spaces $\left[H^{k}(\Omega)\right]^{m}$ and $\left[L^{p}(\Omega)\right]^{m}$ are written as $\mathcal{H}^{k}$ and $\mathcal{L}^{p}$ respectively and we generally omit dependence on $\Omega$ in notation of these spaces. $|\Omega|$ denotes the Lebesgue measure of $\Omega$. Because of the semigroup approach only the time argument of the solution $w$ is explicitly distinguished. On the other hand, the integration is usually taken with respect to $x \in \Omega$, hence throughout the paper all integrals left unspecified should be understood to be taken over $\Omega$, also all constants mentioned are positive numbers.

Let us specify the additional conditions that are required of the function $\Phi$ staying under the gradient on the right side of (1). We claim (besides the $C^{3+\text { Lipschitz }}$ regularity mentioned at the beginning of this paper) that $\Phi$ fulfills the following growth conditions:

$$
\begin{gathered}
\exists_{M \in \mathbb{R}} \forall_{s \in \mathbb{R}^{m}} \Phi(s) \geqslant M, \\
-s^{T}\left(\nabla_{s} \Phi(s)\right) \leqslant-k_{0}|s|^{2 p}+k_{1}, \quad s \in \mathbb{R}^{m}, \\
\left|\frac{\partial^{2} \Phi}{\partial s_{i} \partial s_{j}}(s)\right| \leqslant k_{2}\left(1+|s|^{2 p-2}\right), \quad 1 \leqslant i, j \leqslant m, \quad s \in \mathbb{R}^{m}, \\
\left|\frac{\partial^{3} \Phi}{\partial s_{i} \partial s_{j} \partial s_{l}}(s)\right| \leqslant k_{3}\left(1+|s|^{2 p-3}\right), \quad 1 \leqslant i, j, l \leqslant m, \quad s \in \mathbb{R}^{m}, \\
\left|\nabla_{s} \Phi(s)\right| \leqslant k_{4}|s|^{2 p-1}+k_{4}^{\prime}, \quad s \in \mathbb{R}^{m},
\end{gathered}
$$

and additionally, everywhere in (4)-(8) we require $p=2$ if $n=3$.

REMARK 1. As a simple application of the Main Value Theorem it may be seen that the 
assumption (6) is a consequence of (7) and, in order, (8) is a consequence of (6). Therefore (7) is the only necessary assumption among (6)-(8), and we decided to formulate (6) and (8) explicitly only for simplicity of further references.

REMARK 2. Although conditions (4)-(8) seem to be complicated, there are many functions satisfying all of them. As a "model function" which satisfies (4)-(8), we can take the real polynomial of order $2 p$, having all leading coefficients positive; that is a function of the variable $s=\left(s_{1}, \ldots, s_{m}\right)$ of the form:

$$
\Phi(s)=\sum_{i=1}^{m} a_{i}^{2} s_{i}^{2 p}+(\text { a polynomial of order } \leqslant 2 p-1)
$$

However, our $\Phi$ need not to be a polynomial function as are those considered in [10] or [2]. Moreover, the lower bound (5) will be used in our paper only in the proof of Lemma 5 which offers the possibility of further generalisations. For example, condition (5) can be omitted in the case when $\Phi$ is convex, that is, the Hessian $\Phi^{\prime \prime}$ is nonnegative definite (compare Remark 4 below).

2. System (1)-(3) as a Cauchy problem in a Banach space. Existence of a global SOlution

Let us write (1)-(3) as an evolution problem

$$
\left\{\begin{array}{l}
\dot{w}+A w=F(w), \quad t>0 \\
w(0)=w_{0}
\end{array}\right.
$$

where $F(w)=\Delta \nabla_{w} \Phi(w)$ and the operator $A=\Gamma \Delta^{2}$ is considered on the Hilbert space $\mathcal{L}^{2}$ with dense domain

$$
D(A)=\left\{\phi \in \mathcal{H}^{4} ; \nabla_{x} \phi N=\nabla_{x}(\Delta \phi) N=0 \text { on } \partial \Omega\right\}
$$

In order to use the existence theory developed in [6] with the improvements mentioned in [5, p.73], we shall show that $A$ is sectorial, whereas the nonlinear term $F$ is locally Lipschitz continuous as the operation between the spaces $D\left(A^{1 / 2}\right)=\left\{\phi \in \mathcal{H}^{2} ; \nabla_{x} \phi N=\right.$ 0 on $\partial \Omega\}$ and $\mathcal{L}^{2}$.

Denoting by $\langle\cdot, \cdot\rangle$ the scalar product in $\mathcal{L}^{2}$ and defining

$$
\mathcal{A}:=A+\delta_{0} \Gamma
$$


where $\delta_{0}>0$ is taken so large that $\left(\Delta^{2}+\delta_{0}\right)$ is an isomorphism between $D(A)$ and $\mathcal{L}^{2}$ (see [11, Theorem 5.5.1]), we find for any $\phi, \psi \in D(A)$, that

$$
\begin{aligned}
\langle\mathcal{A} \phi, \psi\rangle & =\int \psi^{T} \Gamma \Delta^{2} \phi d x+\delta_{0} \int \psi^{T} \Gamma \phi d x \\
& =-\int \operatorname{tr}\left(\nabla_{x} \psi^{T} \Gamma \nabla_{x}(\Delta \phi)\right) d x+\delta_{0} \int \psi^{T} \Gamma \phi d x \\
& =\int \Delta \psi^{T} \Gamma \Delta \phi d x+\delta_{0} \int \psi^{T} \Gamma \phi d x .
\end{aligned}
$$

Since $\Gamma$ is symmetric we have analogously from (11) that

$$
\begin{aligned}
\int \Delta \psi^{T} \Gamma \Delta \phi d x+\delta_{0} \int \phi^{T} \Gamma \psi d x & =\int \Delta^{2} \psi^{T} \Gamma \phi d x+\delta_{0} \int \psi^{T} \Gamma \phi d x \\
& =\langle\phi, \mathcal{A} \psi\rangle,
\end{aligned}
$$

which proves the symmetry of $\mathcal{A}$. Next, since $\Gamma$ is positive definite, the operator $\mathcal{A}$ is bounded below, that is, for each $\phi \in D(A)$

$$
\begin{aligned}
\langle\mathcal{A} \phi, \phi\rangle & =\int \phi^{T} \Gamma \Delta^{2} \phi d x+\delta_{0} \int \phi^{T} \Gamma \phi d x \\
& =\int \Delta \phi^{T} \Gamma \Delta \phi d x+\delta_{0} \int \phi^{T} \Gamma \phi d x \geqslant \delta_{0}\langle\phi, \phi\rangle .
\end{aligned}
$$

Moreover, thanks to the choice of $\delta_{0}$, we have

$$
D(A) \underset{\text { isomorphism }}{\stackrel{\Delta^{2}+\delta_{0}}{\rightarrow}} \mathcal{L}^{2} \underset{\text { isomorphism }}{\stackrel{\Gamma}{\longrightarrow}} \mathcal{L}^{2},
$$

so that the range of $\mathcal{A}$ is the whole space $\mathcal{L}^{2}$. From these observations it is clear that $\mathcal{A}$ is self-adjoint and bounded below and hence, in particular, sectorial. Then using [6, Exercise 6 or Theorem 1.3.2] we obtain finally that $A$ is itself sectorial.

The second property we should investigate is the local Lipschitz continuity of the nonlinear function $F$ standing on the right side in (9), that is, we need to check the following condition

$$
\underset{\phi, \psi \in U \subset D\left(A^{1 / 2}\right)}{\forall}\left\|\Delta \nabla_{\phi} \Phi(\phi)-\Delta \nabla_{\psi} \Phi(\psi)\right\|_{\mathcal{L}^{2}} \leqslant k_{U}\|\phi-\psi\|_{D\left(A^{1 / 2}\right)} .
$$

We find by differentiation that

$$
\begin{aligned}
& \Delta \frac{\partial \Phi}{\partial \phi_{i}}(\phi)-\Delta \frac{\partial \Phi}{\partial \psi_{i}}(\psi)=\sum_{l=1}^{m}\left(\frac{\partial^{2} \Phi}{\partial \psi_{l} \partial \phi_{i}}(\phi) \Delta \phi_{l}-\frac{\partial^{2} \Phi}{\partial \psi_{l} \partial \psi_{i}}(\psi) \Delta \psi_{l}\right) \\
& +\sum_{k, l=1}^{m}\left(\frac{\partial^{3} \Phi}{\partial \phi_{k} \partial \phi_{l} \partial \phi_{i}}(\phi)\left(\nabla \phi_{k} \nabla \phi_{l}\right)-\frac{\partial^{3} \Phi}{\partial \psi_{k} \partial \psi_{l} \partial \psi_{i}}(\psi)\left(\nabla \psi_{k} \nabla \psi_{l}\right)\right)
\end{aligned}
$$


Transforming the right side of (15) and taking $L^{2}$ norms of both sides we obtain further (16)

$$
\begin{aligned}
& \left\|\Delta \frac{\partial \Phi}{\partial \phi_{i}}(\phi)-\Delta \frac{\partial \Phi}{\partial \psi_{i}}(\psi)\right\|_{L^{2}} \leqslant \max _{1 \leqslant i, l \leqslant m}\left\|\frac{\partial^{2} \Phi}{\partial \psi_{l} \partial \psi_{i}}(\psi)\right\|_{L^{\infty}} \sum_{l=1}^{m}\left\|\Delta \phi_{l}-\Delta \psi_{l}\right\|_{L^{2}} \\
& \quad+\max _{1 \leqslant i, l \leqslant m}\left\|\frac{\partial^{2} \Phi}{\partial \phi_{l} \partial \phi_{i}}(\phi)-\frac{\partial^{2} \Phi}{\partial \psi_{l} \partial \psi_{i}}(\psi)\right\|_{L^{\infty}} \sum_{l=1}^{m}\left\|\Delta \phi_{l}\right\|_{L^{2}} \\
& \quad+\max _{1 \leqslant i, k, l \leqslant m}\left\|\frac{\partial^{3} \Phi}{\partial \psi_{k} \partial \psi_{l} \partial \psi_{i}}(\psi)\right\|_{L^{\infty}} \sum_{k, l=1}^{m}\left\|\left(\nabla \phi_{k}-\nabla \psi_{k}\right) \nabla \phi_{l}\right\|_{L^{2}} \\
& \quad+\max _{1 \leqslant i, k, l \leqslant m}\left\|\frac{\partial^{3} \Phi}{\partial \psi_{k} \partial \psi_{l} \partial \psi_{i}}(\psi)\right\|_{L^{\infty}} \sum_{k, l=1}^{m}\left\|\left(\nabla \phi_{l}-\nabla \psi_{l}\right) \nabla \psi_{k}\right\|_{L^{2}} \\
& \quad+\max _{1 \leqslant i, k, l \leqslant m}\left\|\frac{\partial^{3} \Phi}{\partial \phi_{k} \partial \phi_{l} \partial \phi_{i}}(\phi)-\frac{\partial^{3} \Phi}{\partial \psi_{k} \partial \psi_{l} \partial \psi_{i}}(\psi)\right\|_{L^{\infty}} \sum_{k, l=1}^{m}\left\|\nabla \phi_{k} \nabla \phi_{l}\right\|_{L^{2}}
\end{aligned}
$$

Remember that $\phi, \psi$ are taken from a bounded set $U \subset \mathcal{H}^{2}$ and also that Sobolev embeddings $H^{1} \subset L^{4}, H^{2} \subset L^{\infty}$ hold for space dimensions $n \leqslant 3$. Hence since the partial derivatives of $\Phi$ are locally Lipschitz continuous and locally bounded (Lipschitz constants and upper bounds are denoted by common symbols $L_{U}$ and $M_{U}$, respectively), inequality (16) gives:

$$
\begin{gathered}
\left\|\Delta \frac{\partial \Phi}{\partial \phi_{i}}(\phi)-\Delta \frac{\partial \Phi}{\partial \psi_{i}}(\psi)\right\|_{L^{2}} \leqslant M_{U} \sum_{l=1}^{m}\left\|\Delta \phi_{l}-\Delta \psi_{l}\right\|_{L^{2}} \\
+L_{U}\|\phi-\psi\|_{L^{\infty}} \sum_{l=1}^{m}\left\|\Delta \phi_{l}\right\|_{L^{2}}+2 M_{U} \sum_{k, l=1}^{m}\left\|\nabla \phi_{k}-\nabla \psi_{k}\right\|_{L^{4}}\left\|\nabla \phi_{l}\right\|_{L^{4}} \\
+L_{U}\|\phi-\psi\|_{L^{\infty}} \sum_{k, l=1}^{m}\left\|\nabla \phi_{k}\right\|_{L^{4}}\left\|\nabla \phi_{l}\right\|_{L^{4}} \leqslant k_{U}\|\phi-\psi\|_{\mathcal{H}^{2}}
\end{gathered}
$$

where the constant $k_{U}$ depends only on the set $U$. Condition (14) is thus justified.

The general theory of $[6, p .54]$ now guarantees the existence of a local solution for the problem (9), that is, there are a positive time $\tau=\tau\left(w_{0}\right)$ and a function $w:[0, \tau) \rightarrow$ $\mathcal{L}^{2}$ which satisfies (9) for $t<\tau$. Moreover, $w(t)$ belongs to $D(A)$ for $t \in(0, \tau)$ and also

$$
w \in C\left([0, \tau) ; D\left(A^{1 / 2}\right)\right), \Delta \nabla_{w} \Phi(w) \in C\left([0, \tau) ; \mathcal{L}^{2}\right) .
$$

In fact, as will be shown in Section 3.D, the $\mathcal{H}^{2}$ norm of the solution is a priori bounded uniformly for $t \in[0, \infty)$. This property of the solution implies, in particular, global 
boundedness in time of the quotient

$$
\frac{\left\|\Delta \nabla_{w}(\Phi(w))\right\|_{\mathcal{L}^{2}}}{1+\|w(t)\|_{D\left(A^{1 / 2}\right)}}
$$

so that in consequence, according to [6, Exercise 1, p.58], the solution $w$ exists on the whole half-line $[0,+\infty)$. Moreover, the family of operators $\{T(t)\}_{t \geqslant 0}$ defined by $T(t) w_{0}:=w\left(t, w_{0}\right)$ forms a strongly continuous semigroup on the space $D\left(A^{1 / 2}\right)$ (compare $[5$, p.73]).

\section{SOME PROPERTIES OF THE SYSTEM (1)-(3)}

\section{A. Preservation of spatial average of the solution.}

In the physical model described by (1)-(3), preservation of spatial average of the solution corresponds to mass conservation. This property follows immediately by integration of (1) over $x \in \Omega$ and by parts. Because of boundary conditions (2) the integrals over $\partial \Omega$ disappear and we obtain

$$
\frac{d}{d t} \frac{1}{|\Omega|} \int w(t) d x=\frac{d}{d t} \bar{w}(t)=0
$$

or equivalently

$$
\bar{w}(t)=\bar{w}_{0}, \quad t \geqslant 0
$$

B. Existence of the Lyapunov functional.

Multiplying (1) by $\left[-\Gamma \Delta w+\nabla_{w} \Phi(w)\right]^{T}$ we find by integration that

$$
\int\left[-\Gamma \Delta w+\nabla_{w} \Phi(w)\right]^{T} w_{t} d x=-\sum_{i=1}^{m} \int\left|\nabla\left(-\sum_{j=1}^{m} \Gamma_{i j} \Delta w_{j}+\frac{\partial \Phi}{\partial w_{i}}(w)\right)\right|^{2} d x
$$

that is, for all $t>0$ the left side of (19) is non-positive, moreover

$$
0 \geqslant \int\left[-\Gamma \Delta w+\nabla_{w} \Phi(w)\right]^{T} w_{t} d x=\frac{d}{d t}\left(\int \frac{1}{2} \operatorname{tr}\left(\nabla_{x} w^{T} \Gamma \nabla_{x} w\right) d x+\int \Phi(w) d x\right)
$$

Thus we may define the Lyapunov functional $\mathcal{L}$ for $(1)-(3)$ as

$$
\mathcal{L}(w(t))=\int \frac{1}{2} \operatorname{tr}\left(\nabla_{\boldsymbol{x}} w^{T} \Gamma \nabla_{x} w\right) d x+\int \Phi(w) d x
$$

It is clear from (19), (20) that $\mathcal{L}$ decreases along each trajectory. Furthermore: 
LEMмA 1. If for a solution $w$ of the system (1)-(3)

$$
\mathcal{L}(w(t))=\text { constant }, \quad t>0,
$$

then $w$ is a stationary (time independent) solution of (1)-(3).

Proof: Because for each positive $t, w(t)$ belongs to $D(A)$, conditions (22) and (19), (20) ensure that

$$
\forall_{t>0} \forall_{i=1,2 \ldots m}\left|\nabla\left(-\sum_{j=1}^{m} \Gamma_{i j} \Delta w_{j}(t)+\frac{\partial \Phi}{\partial w_{i}}(w(t))\right)\right|^{2}=0 \quad \text { almost everywhere in } \Omega .
$$

Next, from the well known property of distributional derivatives (see $[9, p .92]$ ), the function under the gradient in (23) is independent of $x$. Thus, since $C^{2}(\Omega) \subset D(A)$ for $n \leqslant 3$, condition (23) gives:

$$
\forall_{t>0} \exists_{c(t) \in \mathbb{R}^{m}}-\Gamma \Delta w(t)+\nabla_{w} \Phi(w(t))=c(t) \quad \text { for every } x \in \Omega
$$

Further, taking the Laplacian of both sides in (24) we obtain that

$$
\Delta\left[-\Gamma \Delta w(t)+\nabla_{w} \Phi(w(t))\right]=0, \quad t \in(0,+\infty) .
$$

As $w(t)$ is a solution of (1)-(3), equality (25) implies that

$$
\frac{d w}{d t}(t)=0 \quad \text { for } t>0
$$

therefore $w(t)$ is time independent for $t>0$. Finally, because of $(17), w(t)=w_{0}$ for all $t \geqslant 0$. Lemma 1 is proved.

We use the above considerations in the proof of the following:

LEMMA 2. An element $w \in D(A)$ is a stationary solution of (1)-(3) if and only if $w$ is a $\mathcal{H}^{2}$ solution of the Neumann type elliptic boundary value problem:

$$
\begin{cases}-\Gamma \Delta v+\nabla_{v} \Phi(v)=a & \text { in } \Omega \\ \nabla_{x} v N=0 & \text { on } \partial \Omega\end{cases}
$$

Proof: Based on the considerations leading to (24), it is clear that if $w$ is a stationary solution of (1)-(3) then, in particular, $w$ is a solution of the elliptic problem (26) with some $a \in \mathbb{R}^{m}$. Moreover, by integrating (26) over $x \in \Omega$, it is possible to determine explicitly the constant vector $a$ as:

$$
a=\overline{\nabla_{v} \Phi(v)}
$$


For the converse, if $v \in D\left(A^{1 / 2}\right)$ solves

$$
-\Gamma \Delta v+\nabla_{v} \Phi(v)=a
$$

then from elliptic regularity theory $[8], v \in \mathcal{H}^{4}$ and also:

$$
\nabla_{x}(\Delta v) N=\nabla_{x}\left(\Gamma^{-1}\left(-\nabla_{v} \Phi(v)+a\right)\right)=0 \quad \text { on } \partial \Omega
$$

Thus $v \in D(A)$, whereas taking Laplician of both sides in (28)

$$
\Delta\left(-\Gamma \Delta v+\nabla_{v} \Phi(v)\right)=0
$$

so that $w(t)=v$ for all $t \geqslant 0$ is a time independent solution of (1)-(3) starting from $w(0)=v$. The proof is completed.

C. Global $\mathcal{H}^{1}$ estimate of the solution.

Existence of the Lyapunov functional $\mathcal{L}$ guarantees a global in time estimate of the norm $\left\|w_{i}\right\|_{H^{1}}$ for each component $w_{i}$ of the solution $w$. Since $\Phi$ is bounded below (condition (4)) and $\mathcal{L}$ decreases along each trajectory, we have from (21):

$$
\int \frac{1}{2} \operatorname{tr}\left(\nabla_{x} w^{T} \Gamma \nabla_{x} w\right) d x \leqslant \mathcal{L}\left(w_{0}\right)-\int \Phi(w) d x \leqslant \mathcal{L}\left(w_{0}\right)-M|\Omega| .
$$

Next, since $\Gamma$ is positive definite, then

$$
\int \frac{1}{2} \operatorname{tr}\left(\nabla_{x} w^{T} \Gamma \nabla_{x} w\right) d x \geqslant \gamma_{0} \sum_{i=1}^{m}\left\|\nabla w_{i}\right\|_{L^{2}}^{2}
$$

Finally, as the expression

$$
\left(\|\nabla \phi\|_{\mathcal{L}^{2}}^{2}+|\bar{\phi}|^{2}\right)^{1 / 2}
$$

defines on $D\left(A^{1 / 2}\right)$ the norm equivalent to the natural $\mathcal{H}^{1}$ norm, then collecting estimates (18), (30), (31) we reach the required property

$$
\|w(t)\|_{\mathcal{H}^{1}} \leqslant \text { constant }
$$

with constant independent of $t \in[0,+\infty)$.

D. Global $\mathcal{H}^{2}$ estimate of the solution.

To obtain global in time boundedness of $\|w\|_{\mathcal{H}^{2}}$ it is necessary to find first a suitable estimate for the nonlinear term $\Delta \nabla_{w} \Phi(w)$. Hence, we start with the following auxiliary inequality, which is valid (with some $k>0$ ) only on a solution $w(t)$ of the system (1)-(3); 
LEмma 3. There exists a positive constant $k=k\left(\left\|w_{0}\right\|_{\mathcal{H}^{1}}, \Omega\right)$ such that for a solution $w(t)$ of the system (1)-(3):

$$
\left\|\Delta \nabla_{w} \Phi(w(t))\right\|_{\mathcal{L}^{2}}^{2} \leqslant k\left(1+\left\|\Delta^{2} w(t)\right\|_{C^{2}}^{4 / 3}\right), \quad t \geqslant 0
$$

PROOF: Condition (34) stated above is similar to inequality (4.105) developed in $[10$, p.156], thus to derive (34) we shall follow the concept of [10].

Estimating the $i$-th component $\Delta \frac{\theta \Phi}{\partial w_{i}}(w)$ of the nonlinear term $\Delta \nabla_{w} \Phi(w)$ we have

$$
\begin{aligned}
\left|\Delta \frac{\partial \Phi}{\partial w_{i}}(w)\right|= & \left|\sum_{l, j=1}^{m} \sum_{k=1}^{n} \frac{\partial^{3} \Phi}{\partial w_{j} \partial w_{l} \partial w_{i}}(w) \frac{\partial w_{l} \partial w_{j}}{\partial x_{k} \partial x_{k}}+\sum_{j=1}^{m} \frac{\partial^{2} \Phi}{\partial w_{j} \partial w_{i}}(w) \Delta w_{j}\right| \\
\leqslant & \max _{1 \leqslant j, l, i \leqslant m}\left|\frac{\partial^{3} \Phi}{\partial w_{j} \partial w_{l} \partial w_{i}}(w)\right| \sum_{l, j=1}^{m}\left|\nabla\left(w_{l}\right) \nabla\left(w_{j}\right)\right| \\
& +\max _{1 \leqslant i, j \leqslant m}\left|\frac{\partial^{2} \Phi}{\partial w_{j} \partial w_{i}}(w)\right| \sum_{j=1}^{m}\left|\Delta w_{j}\right| .
\end{aligned}
$$

Taking now $L^{2}$ norms of both sides of inequality (35) and using assumptions (6), (7) we obtain

$$
\begin{aligned}
\left\|\Delta \frac{\partial \Phi}{\partial w_{i}}(w)\right\|_{L^{2}} \leqslant & k_{3}\left(1+\|w\|_{L^{\infty}}^{2 p-3}\right) \sum_{l, j=1}^{m}\left\|\nabla\left(w_{l}\right) \nabla\left(w_{j}\right)\right\|_{L^{2}} \\
& +k_{2}\left(1+\|w\|_{L^{\infty}}^{2 p-2}\right) \sum_{j=1}^{m}\left\|\Delta w_{j}\right\|_{L^{2}} \\
\leqslant & k^{\prime}\left[\left(1+\sum_{j=1}^{m}\left\|w_{j}\right\|_{L^{\infty}}^{2 p-3}\right) \sum_{j=1}^{m}\left\|\nabla\left(w_{j}\right)\right\|_{L^{4}}^{2}\right. \\
& \left.+\left(1+\sum_{j=1}^{m}\left\|w_{j}\right\|_{L^{\infty}}^{2 p-2}\right) \sum_{j=1}^{m}\left\|\Delta w_{j}\right\|_{L^{2}}\right] .
\end{aligned}
$$

We are now in the position fully analogous to [10, condition (4.109), p.157]. If for each particular component $w_{j}$ we follow the considerations of [10, between $\left.(4.110)-(4.116)\right]$, we find similarly as in $[\mathbf{1 0}]$ that:

$$
\left\{\begin{array}{l}
\left\|\Delta w_{j}\right\|_{L^{2}} \leqslant k_{5}\left\|\Delta^{2} w_{j}\right\|_{L^{2}}^{1 / 3} \\
\left\|\nabla w_{j}\right\|_{L^{4}} \leqslant k_{6}\left\|\Delta w_{j}\right\|_{L^{2}}^{n / 12},
\end{array}\right.
$$


and further, depending on the space dimension $n$,

$$
\begin{cases}\left\|w_{j}-\bar{w}_{0 j}\right\|_{L^{\infty}} \leqslant k_{7}\left\|\nabla w_{j}\right\|_{L^{2}} & \text { for } n=1, \\ \left\|w_{j}-\bar{w}_{0 j}\right\|_{L^{\infty}} \leqslant k_{8}\left\|\nabla w_{j}\right\|_{L^{2}}^{(1-e)}\left\|\Delta^{2} w_{j}\right\|_{L^{2}}^{e} & \text { for } n=2 \text { and any } \varepsilon>0 \\ \left\|w_{j}-\bar{w}_{0 j}\right\|_{L^{\infty}} \leqslant k_{9}\left\|\nabla w_{j}\right\|_{L^{2}}^{5 / 6}\left\|\Delta^{2} w_{j}\right\|_{L^{2}}^{1 / 6} & \text { for } n=3\end{cases}
$$

Inserting (37) and (38) into (36), using the global $\mathcal{H}^{1}$ boundedness of $w(t)$ (shown in Section 3.C) and applying an obvious inequality

$$
\forall a>a^{\prime}>0 \forall_{b} \geqslant 0 b^{1 / a} \leqslant 1+b^{1 / a^{\prime}}
$$

we come finally to (34) with the constant $k$ dependent on the $\mathcal{H}^{1}$ norm of the initial function $w_{0}$. Condition (34) is justified.

We are now able to prove the following:

LEMMA 4. If $w$ is a solution of (1)-(3), then for all $t \geqslant 0$ :

$$
\|\Delta w(t)\|_{\mathcal{L}^{2}}^{2} \leqslant \max \left\{k_{8} z_{1},\left\|\Delta w_{0}\right\|_{\mathcal{L}^{2}}^{2}\right\}
$$

where constants $k_{8}, z_{1}$ are given in (45), (46) respectively. Moreover, since the norm

$$
\left(\|\Delta \phi\|_{\mathcal{L}^{2}}^{2}+|\bar{\phi}|^{2}\right)^{1 / 2}
$$

on $D\left(A^{1 / 2}\right)$ is equivalent to the natural $\mathcal{H}^{2}$ norm, then because of the mass conservation property shown in Section 3.A:

$$
\|w(t)\|_{\mathcal{H}^{2}} \leqslant \text { constant }\left(\left\|w_{0}\right\|_{\mathcal{H}^{2}}, \Omega\right), \quad t \geqslant 0
$$

PROOF: By multiplying (1) by $\Delta^{2} w^{T}$ and integrating over $\Omega$ we obtain:

$$
\int \Delta^{2} w^{T} w_{t} d x=-\int \Delta^{2} w^{T} \Gamma \Delta^{2} w d x+\int \Delta^{2} w^{T} \Delta\left(\nabla_{w} \Phi(w)\right) d x
$$

Next, since $\Gamma$ is positive definite, the Hölder and Cauchy inequalities give:

$$
\begin{aligned}
\frac{1}{2} \frac{d}{d t}\|\Delta w\|_{\mathcal{L}^{2}}^{2} & \leqslant-\gamma_{0}\left\|\Delta^{2} w\right\|_{\mathcal{C}^{2}}^{2}+\left\|\Delta^{2} w\right\|_{\mathcal{L}^{2}}\left\|\Delta\left(\nabla_{w} \Phi(w)\right)\right\|_{\mathcal{L}^{2}} \\
& \leqslant-\frac{\gamma_{0}}{2}\left\|\Delta^{2} w\right\|_{\mathcal{L}^{2}}^{2}+\frac{1}{2 \gamma_{0}}\left\|\Delta\left(\nabla_{w} \Phi(w)\right)\right\|_{\mathcal{L}^{2}}^{2}
\end{aligned}
$$

Inserting (34) into (43) we have

$$
\frac{d}{d t}\|\Delta w(t)\|_{\mathcal{L}^{2}}^{2} \leqslant-\gamma_{0}\left\|\Delta^{2} w(t)\right\|_{\mathcal{L}^{2}}^{2}+\frac{k}{\gamma_{0}}\left(1+\left(\left\|\Delta^{2} w(t)\right\|_{\mathcal{L}^{2}}^{2}\right)^{2 / 3}\right) .
$$


Let us note further, that as a consequence of the boundary conditions (2), $\int \Delta w(t) d x=$ 0 and hence, according to the Smoller inequality $([7, \mathrm{p} .112])$, we have

$$
\|\Delta w\|_{\mathcal{L}^{2}}^{2} \leqslant k_{8}\left\|\Delta^{2} w\right\|_{\mathcal{L}^{2}}^{2} .
$$

We define the constant $z_{1}$ as the unique positive solution of the algebraic equation:

$$
-\gamma_{0} z+\frac{k}{\gamma_{0}}\left(1+z^{2 / 3}\right)=0
$$

As a consequence of (45), whenever $\|\Delta w(t)\|_{L^{2}}^{2}>k_{8} z_{1}$ then, by the definition of $z_{1}$, the right side of the differential inequality (44) is negative, hence $\|\Delta w(t)\|_{\mathcal{L}^{2}}^{2}$ needs to decrease. This shows (compare [2, Lemma 5] for detailed proof), that (39) is satisfied. The proof of Lemma 4 is completed.

REMARK 3. To justify the correctness of the calculations in Lemma 4 we need additional smoothness of the solution $w$. As a result of $[6$, Theorem 3.5.2], the function $(0, \infty) \ni$ $t \longrightarrow \dot{w}(t) \in D\left(A^{1 / 2}\right)$ is continuous. Then, as a consequence of (17) and (9), also the function $(0, \infty) \ni t \longrightarrow A w(t) \in \mathcal{L}^{2}$ is continuous and one can check that for $t>0$ :

$$
\left|h^{-1}\left(\int|\Delta w(t+h)|^{2} d x-\int|\Delta w(t)|^{2} d x\right)-2 \int \Delta^{2} w^{T} w_{t} d x\right| \longrightarrow 0 \text { as } h \rightarrow 0^{+}
$$

or

$$
\frac{1}{2} \frac{d}{d t} \int|\Delta w(t)|^{2} d x=\int \Delta^{2} w^{T} w_{t} d x \text { for } t>0 .
$$

E. Boundedness of the SET OF STATIONARY SOlutions.

Let us denote by $\mathcal{D}$ the subset of the space $D\left(A^{1 / 2}\right)$ consisting of all fixed points of the semigroup $\{T(t)\}_{t \geqslant 0}$, that is, of all stationary solutions of the system (1)-(3) (the symbols $D\left(A^{1 / 2}\right)$ and $\{T(t)\}_{t \geqslant 0}$ were introduced in Section 2). According to the considerations of Section 3.B (Lemma 2), the following characterisation holds (remembering that $\bar{\phi}$ is the spatial average of $\phi$ over $\Omega$ ):

$$
\mathcal{D}=\left\{v \in D\left(A^{1 / 2}\right) ;-\Gamma \Delta v+\nabla_{v} \Phi(v)=a, \quad a \in \mathbb{R}^{m}, \quad a=\overline{\nabla_{v} \Phi(v)}\right\}
$$

Then for fixed $\alpha>0$, let $\mathcal{D}_{\alpha}$ be the subset of $\mathcal{D}$ consisting of all elements with $|\bar{v}|$ not exceeding $\alpha$. In the next section boundedness of the set of stationary solutions will play a crucial role in the construction of the global attractor. Thus we shall prove:

Lemma 5. For each $\alpha>0, D_{\alpha}$ is a bounded subset of $D\left(A^{1 / 2}\right)$.

Proof: Since an element $v$ of $\mathcal{D}$ is characterised in (47) as a solution of a Neumann type elliptic boundary value problem, then we have

$$
\int v^{T}\left[-\Gamma \Delta v+\nabla_{v} \Phi(v)\right] d x=\int v^{T} a d x
$$


Further, in the presence of the definition of $a$ (stated in (47)), since $\Gamma$ is positive definite (48) gives

$$
\gamma_{0} \sum_{i=1}^{m} \int\left|\nabla v_{i}\right|^{2} d x \leqslant \int \bar{v}^{T} \nabla_{v} \Phi(v) d x-\int v^{T} \nabla_{v} \Phi(v) d x
$$

Next, it follows from (8) and the Young inequality that

$$
\forall_{\nu>0} \exists_{C(\nu)>0} \forall_{s \in \mathbb{R}^{m}}\left|\nabla_{s} \Phi(s)\right| \leqslant \nu|s|^{2 p}+C(\nu) .
$$

Hence, applying to the right side of (49) the Schwarz inequality and growth conditions (5), (50), we obtain:

$$
\begin{gathered}
\gamma_{0} \sum_{i=1}^{m} \int\left|\nabla v_{i}\right|^{2} d x \leqslant \int\left|\nabla_{v} \Phi(v)\right||\bar{v}| d x-k_{0} \int|v|^{2 p} d x+k_{1}|\Omega| \\
\leqslant\left(\nu|\bar{v}|-k_{0}\right) \int|v|^{2 p} d x+\left(C(\nu)|\bar{v}|+k_{1}\right)|\Omega| .
\end{gathered}
$$

By substituting in (51) $\nu=\nu_{0}=\left(k_{0}\right) /(|\bar{v}|+1)$ in order to obtain a negative coefficient before $\int|v|^{2 p} d x$, we reach the estimate

$$
\gamma_{0} \sum_{i=1}^{m} \int\left|\nabla v_{i}\right|^{2} d x \leqslant\left(C\left(\nu_{0}\right)|\bar{v}|+k_{1}\right)|\Omega|
$$

where the right side depends only on given quantities. Moreover, for the equivalent norm (32) on $\mathcal{H}^{1}$, condition (52) ensures that

$$
\|v\|_{\mathcal{H}^{1}} \leqslant k_{9}(|\bar{v}|,|\Omega|) .
$$

The inequality (53) is crucial for the rest of the proof. Using once again the characterisation of the elements of $\mathcal{D}$ (see (47)) we find:

$$
\int \Delta v^{T}\left[-\Gamma \Delta v+\nabla_{v} \Phi(v)\right] d x=\int \Delta v^{T} a d x=0
$$

Since the matrix $\Gamma$ is positive definite, equality (54) gives

$$
-\gamma_{0} \int|\Delta v|^{2} d x+\left(\int\left|\nabla_{v} \Phi(v)\right|^{2} d x\right)^{1 / 2}\left(\int|\Delta v|^{2} d x\right)^{1 / 2} \geqslant 0
$$

and further (from the Cauchy inequality),

$$
\int|\Delta v|^{2} d x \leqslant \frac{1}{\gamma_{0}^{2}} \int\left|\nabla_{v} \Phi(v)\right|^{2} d x
$$


Now, based on the growth condition (8), we can increase the right side of (56) coming to

$$
\int|\Delta v|^{2} d x \leqslant \frac{1}{\gamma_{0}^{2}} \int\left(k_{4}|v|^{2 p-1}+k_{4}^{\prime}\right)^{2} d x \leqslant \frac{2 k_{4}^{2}}{\gamma_{0}^{2}} \int|v|^{4 p-2} d x+\frac{2 k_{4}^{\prime 2}|\Omega|}{\gamma_{0}^{2}}
$$

In the presence of the Sobolev embeddings (remembering that $p=2$ if $n=3$ ) the right side of (57) is estimated by ( constant $_{1}\|v\|_{\mathcal{H}^{1}}^{4 p-2}+$ constant $_{2}$ ). Moreover, since inequality (53) assures boundedness of $\|v\|_{\mathcal{H}^{1}}$ only in terms of $|\bar{v}|$ and $|\Omega|$, formula (57) gives immediately:

$$
\|\Delta v\|_{\mathcal{L}^{2}} \leqslant k_{10}(|\bar{v}|, \Omega)
$$

Then considering the equivalent norm (40) on $D\left(A^{1 / 2}\right)$, we obtain finally that every element $v$ from $\mathcal{D}_{\alpha}$ satisfies:

$$
\|v\|_{\mathcal{H}^{2}} \leqslant k_{11}(\alpha, \Omega)
$$

Lemma 5 is thus proved.

\section{DisSIPATIVENESS AND GLOBAL ATTRACTOR}

Since the set $\mathcal{D}$ introduced in (47) contains all constant vector functions, that is $\left\{v(x) \equiv \bar{v} ; \bar{v} \in \mathbb{R}^{m}\right\} \subset \mathcal{D}$, it is impossible to construct the global attractor on the whole space $D\left(A^{1 / 2}\right)$. To overcome this difficulty, for fixed $\alpha>0$ we introduce the complete metric space $\mathcal{H}_{\alpha}$ :

$$
\mathcal{H}_{\alpha}:=\left\{u \in D\left(A^{1 / 2}\right) ;|\bar{u}| \leqslant \alpha\right\}
$$

According to the mass conservation property (18) the set $\mathcal{H}_{\alpha}$ is positively invariant, hence we shall consider further $\{T(t)\}_{t \geqslant 0}$ restricted to the semigroup on $\mathcal{H}_{\alpha}$. With the use of our previous results we shall justify that:

LEMMA 6. There exists a bounded subset $\mathcal{B}$ of $\mathcal{H}_{\alpha}$ attracting each point of $\mathcal{H}_{\alpha}$ (that is, the semigroup $\{T(t)\}_{t \geqslant 0}$ on $\mathcal{H}_{\alpha}$ is point dissipative $[5, \mathrm{p} .38]$ ).

Proof: Let us define the set $\mathcal{B}$ as

$$
\mathcal{B}:=\bigcup_{u \in \mathcal{H}_{\alpha}} \omega(u)
$$

where $\omega(u)$ denotes the $\omega$-limit set of $u$. Because of the estimates (18), (41) $\{T(t)\}_{t \geqslant 0}$ takes bounded sets into bounded sets. Moreover, since the operator $A$ (defined below 
formula (9)) is sectorial and, according to [11, Theorem 5.5.1.b] its resolvent is compact, then $\left[5\right.$, Theorem 4.2.2] ensures that $\{T(t)\}_{t \geqslant 0}$ is compact. Hence $\mathcal{B}$ attracts each point of $\mathcal{H}_{\alpha}$, and to be able to use [5, Theorem 4.2.4] we only need to show that $\mathcal{B}$ is bounded in $\mathcal{H}_{\boldsymbol{\alpha}}$.

For every $u_{0} \in \mathcal{B}$ there is $w_{0} \in \mathcal{H}_{\alpha}$ such that $u_{0}$ belongs to $\omega\left(w_{0}\right)$. Then by substituting $w:=T(t) w_{0}$ as an argument of the Lyapunov functional $\mathcal{L}(21)$, it is obvious that:

$$
\exists_{\beta \in \mathbb{B}} \lim _{t \rightarrow+\infty} \mathcal{L}\left(T(t) w_{0}\right)=\beta,
$$

since $\mathcal{L}\left(T(t) w_{0}\right)$ is decreasing and bounded below. Moreover, from a characterisation of $\boldsymbol{\omega}$-limit sets the Lyapunov functional $\mathcal{L}$ is identically equal to $\beta$ along the trajectory of every element from $\omega\left(w_{0}\right)$. Thus we have in particular:

$$
\mathcal{L}\left(T(t) u_{0}\right)=\beta \quad \text { for all } t \geqslant 0,
$$

so that, according to Lemma 1 (Section 3.B), $u_{0}$ must be a stationary solution of the system (1)-(3) belonging to $\mathcal{D}_{\alpha}$. Hence, due to Lemma $5, \mathcal{B}$ is bounded in $D\left(A^{1 / 2}\right)$. The proof of Lemma 6 is finished.

The results we have obtained so far justify the validity of all the required assumptions of [5, Theorem 4.2.4]. As a direct consequence of this theorem we obtain the existence of the global attractor for the system (1)-(3); so we are able to formulate:

Theorem 1. Semigroup $\{T(t)\}_{t \geqslant 0}$ generated by the Cahn-Hilliard system (1)(3) on a metric space $\mathcal{H}_{\alpha}$ possesses a connected global attractor.

\section{ASYMPTOTIC BEHAVIOUR OF THE TRAJECTORIES}

We have already mentioned in Lemma 6 that each element $w_{0}$ of the phase space $D\left(A^{1 / 2}\right)$ is attracted by its $\omega$-limit set $\omega\left(w_{0}\right)$. It has also been shown explicitly that $\omega$-limit sets contain only stationary solutions of the system (1)-(3). However, if the gradient $\nabla \Phi$ is a monotone operator, then any $\omega$-limit set consists of a single element, and in consequence $T(t) w_{0}$ must tend to a stationary solution. Thus let us introduce the set $\mathcal{W} \subset \mathbb{R}^{m}$ :

$$
\mathcal{W}:=\left\{s^{\prime} \in \mathbb{R}^{m} ; \forall_{s \in \mathbb{R}^{m}}\left(s^{\prime}-s\right)^{T}\left(\nabla, \Phi\left(s^{\prime}\right)-\nabla . \Phi(s)\right) \geqslant 0\right\}
$$

We shall prove the following:

THEOREM 2. For each element $w_{0}$ from $D\left(A^{1 / 2}\right)$ with the average $\bar{w}_{0}$ belonging to $\mathcal{W}, T(t) w_{0} \rightarrow \bar{w}_{0}$ in $\mathcal{H}^{2}$ as $t$ goes to infinity.

PROOF: It suffices to show that for arbitrary $w_{0} \in D\left(A^{1 / 2}\right)$ with $\bar{w}_{0} \in \mathcal{W}$, the set $\omega\left(w_{0}\right)$ consists of a single element $\bar{w}_{0}$. If $v \in \omega\left(w_{0}\right)$, then $v$ has the same spatial 
average as $w_{0}$. Moreover, since $v$ and $\bar{w}_{0}$ are time independent solutions of (1)-(3), then with the use of Lemma 2 we find that

$$
-\Gamma \Delta\left(\bar{w}_{0}-v\right)+\nabla_{\bar{w}_{0}} \Phi\left(\bar{w}_{0}\right)-\nabla_{v} \Phi(v)=\overline{\nabla_{\bar{w}_{0}} \Phi\left(\bar{w}_{0}\right)}-\overline{\nabla_{v} \Phi(v)} .
$$

Multiplying (65) in $\mathcal{L}^{2}$ by $\bar{w}_{0}-v$, we obtain ( $\Gamma$ is positive definite):

$$
\begin{aligned}
\gamma_{0} \sum_{i=1}^{m} \int\left|\nabla\left(\bar{w}_{0}-v\right)\right|^{2} d x \leqslant-\int\left(\bar{w}_{0}-v\right)^{T}\left(\nabla_{\bar{w}_{0}} \Phi\left(\bar{w}_{0}\right)-\nabla_{v} \Phi(v)\right) d x \\
\quad+\int\left(\bar{w}_{0}-v\right)^{T}\left(\overline{\nabla_{\bar{w}_{0}} \Phi\left(\bar{w}_{0}\right)}-\overline{\nabla_{v} \Phi(v)}\right) d x \\
=-\int\left(\bar{w}_{0}-v\right)^{T}\left(\nabla_{\bar{w}_{0}} \Phi\left(\bar{w}_{0}\right)-\nabla_{v} \Phi(v)\right) d x .
\end{aligned}
$$

Since $\bar{w}_{0} \in \mathcal{W}$, condition (66) gives:

$$
\sum_{i=1}^{m} \int\left|\nabla\left(\bar{w}_{0}-v\right)\right|^{2} d x=0 .
$$

Then because the spatial average of $v$ is equal to $\bar{w}_{0}$ and $v \in D\left(A^{1 / 2}\right) \subset C^{0}(\bar{\Omega})$, the function $v$ must be identically equal to $\bar{w}_{\mathbf{0}}$. The proof is completed.

REMARK 4. In order to ensure that $\mathcal{W}$ is a nontrivial set, further assumptions on the term $\Phi$ are needed, for example it is clear that $\mathcal{W}=\mathbb{R}^{m}$ if we assume that the function $\Phi$ is convex (that is, its Hessian matrix $\Phi^{\prime \prime}$ is nonnegative definite). However, the set $\mathcal{W}$ can be "large" in $\mathbb{R}^{m}$ also in the case when some special forms of nonlinearities in (1) are considered. For example, let us take

$$
\Phi\left(s_{1}, \ldots, s_{m}\right)=\sum_{i=1}^{m} W_{i}\left(s_{i}\right)
$$

where $W_{i} ; i=1, \ldots, m$ are real polynomials of order $2 p$ with positive leading coefficients (compare the conditions (4)-(8)). For such $W_{i}$ the derivatives $W_{i}^{\prime}(r)$ are monotone for $|r|$ sufficiently large, $i=1, \ldots, m$, so we shall denote by $a_{i}\left(b_{i}\right)$ the value of largest local maximum (smallest local minimum) of $W_{i}^{\prime}$. Thus the set $\mathcal{W}$ consists of all $s^{\prime} \in \mathbb{R}^{m}$ those components $s_{i}^{\prime}$ are outside the intervals $\left(z_{1}^{i}, z_{2}^{i}\right)$ for each $i=1, \ldots, m$, where constants $z_{1}^{i}, z_{2}^{i}$ are defined as

$$
\left\{\begin{array}{l}
z_{1}^{i}=\sup \left\{z ; W_{i}^{\prime}(r) \leqslant b_{i} \text { for all } r \leqslant z\right\}, \\
z_{2}^{i}=\inf \left\{z ; W_{i}^{\prime}(r) \geqslant a_{i} \text { for all } r \geqslant z\right\}, \quad i=1, \ldots, m .
\end{array}\right.
$$




\section{BACKWARD UNIQUENESS RESULT}

At the end of the paper let us summarise some of the results we have obtained so far and consider the semiflow $\{T(t)\}_{t \geqslant 0}$ restricted to the global attractor in the phase space $\mathcal{H}_{\alpha}$. Since for two solutions $w_{1}, w_{2}$ of the problem (1)-(3) the Lipschitz condition (14) holds, then based on the abstract backward uniqueness result stated in [10, Lemma 6.2, p.170] it follows immediately that the restricted $T(t)$ is a one-to-one map for each $t \geqslant 0$. Then using the general semigroup property given in [5, Theorem 3.10, p.56] we obtain finally:

LEMma 7. Semigroup $\{T(t)\}_{t \geqslant 0}$ restricted to the global attractor in the phase space $\mathcal{H}_{\alpha}$ can be extended to a group.

\section{REFERENCES}

[1] J.W. Cahn and J.E. Hilliard, 'Free energy of a nonuniform system, I. Interfacial free energy', J. Chem. Phys. 28 (1958), 258-267.

[2] T. Dlotko, 'Global attractor for the Cahn-Hilliard equation in $H^{2}$ and $H^{3}, J$. Differential Equations (to appear).

[3] C.M. Elliot and S. Luckhaus, 'A generalized diffusion equation for phase separation of a multi-component mixture with interfacial free energy', IMA preprint 887, 1991.

[4] D.J. Eyre, 'Systems of Cahn-Hilliard equations', University of Minnesota, AHPCRC Preprint 92-102, 1992.

[5] J.K. Hale, Asymptotic behavior of dissipative systems (American Mathematical Society, Providence, R.I., 1988).

[6] D. Henry, Geometric theory of semilinear parabolic equations (Springer-Verlag, Berlin, Heidelberg, New York, 1981).

[7] J. Smoller, Shock waves and reaction-diffusion equations (Springer-Verlag, Berlin, Heidelberg, New York, 1988).

[8] V.A. Solonnikov, 'On $L_{p}$ estimates of solutions of elliptic and parabolic systems', Trudy Mat. Inst. Steklov. 102 (1967), 137-160.

[9] Z. Szmydt, Fourier transformation and linear differential equations (D. Reidel Publishing Company (PWN), Dordrecht, Holland, 1977).

[10] R. Temam, Infinite-dimensional dynamical systems in mechanics and physics (SpringerVerlag, Berlin, Heidelberg, New York, 1988).

[11] H. Triebel, Interpolation theory, function spaces, differential operators (Veb Deutscher Verlag, Berlin 1978, also North-Holland, Amsterdam, 1978).

Institute of Mathematics

Silesian University

40-007 Katowice

Poland 